REVISTA IBEROAMERICANA DE EDUCACIÓN A DISTANCIA

Vol. $5 \cdot \mathrm{N}^{\circ} 2$

Diciembre, 2002

\title{
Construcción de cursos hipermedia adaptativos basados en web utilizando AHA
}

\section{(Constuction of Adaptive Hypermedia Web-Based Courses using AHA)}

\author{
CRISTÓBal Romero Morales \\ Sebastián Ventura Soto \\ Carlos de Castro
}

Departamento de Informática y Análisis Numérico

Universidad de Córdoba

(España)

RESUMEN: En el este artículo vamos a presentar una sistema para la construcción de curso hipermedia adaptativos basados en web. Cualquier profesor utilizando nuestro sistema puede crear rápidamente un curso basado en web que además permite la adaptación tanto de la presentación como de la navegación dependiendo del nivel de conocimiento de cada alumno en particular. Para desarrollarlo hemos utilizado de base el sistema adaptativo genérico AHA (de Bra, 98), al que le hemos realizado varias modificaciones importantes para especializarlo y potenciarlo en el área de educación. Como ejemplo de utilización vamos a mostrar un curso de linux que hemos construido utilizando nuestro sistema y que actualmente está siendo utilizado por estudiantes de la Universidad de Córdoba. También estamos desarrollando una herramienta autor para que el profesor pueda construir el curso fácilmente sin la necesidad de conocer la estructura interna de AHA.

Sistemas Hipermedia Adaptativos - AHA - Enseñanza a distancia - Enseñanza Basada en Web Niveles de Conocimiento

ABSTRACT: In this paper we are going to introduce a system to build adaptive hypermedia web-based courses. Using it, any teacher can quickly creates a web-based course that lets the adaptation both the presentation and the navigation depend on the level of each particular student's knowledge. In order to develop it we have used the AHA generic adaptive systems (de Bra, 98). We have performed it several modification on it to specialize and power it in the educational area. We are going to show a linux course as example of using our system. Currently this course is being used by students of the University of Cordoba. The linux course 
Cristóbal Romero Morales, Sebastián Ventura Soto, Carlos de Castro

together with our system can be downloaded from Internet free. We are also developing an author tool so that the teacher can build easily the course without having to know the AHA internal structure.

Adaptive Hypermedia Systems - AHA - Distance learning - Web-based Learning - Levels of Knowledge

\section{INTRODUCCIÓN}

La educación basada en el Web es un área actual de investigación, debido principalmente a sus beneficios como son la independencia de la localización física y de la plataforma. De forma que una aplicación Web instalada en un servidor web puede ser utilizada por miles de estudiantes de todo el mundo con el único requisito de tener una conexión a Internet. Hoy día existen cientos de cursos y aplicaciones educativas basados en web. El problema que tienen estos cursos es que la mayoría de ellos son sólo una red de páginas hipertexto estáticas. Este problema se puede solucionar aumentando la interactividad y la adaptatividad de las aplicaciones (Brusilovsky, 99). La adaptación es especialmente importante debido que a la variedad de usuarios que pueden utilizar la aplicación basada en web es mucho mayor que el de una aplicación de ejecución local. Y una aplicación que ha sido diseñada para una clase particular de usuario en mente, no suele ser útil para otros tipos de usuarios. Los Sistemas Hipermedia Adaptativos construyen un modelo del usuario individual y lo utilizan para adaptar el sistema al usuario, por ejemplo, adaptar el contenido de una página hipermedia al conocimiento u objetivo de un usuario, o sugerirle los enlaces más relevantes a seguir. Una definición de Sistema Hipermedia Adaptativo (Brusilovsky, 96) es: «Por Sistemas Hipermedia Adaptativos queremos decir, todos los sistemas hipertextos e hipermedia que reflejan algunas características del usuario en el modelo del usuario y aplican este modelo en la adaptación de varios aspectos visibles del sistema al usuario». Un buen ejemplo de Sistema Hipermedia Adaptativo genérico es el sistema AHA (Adaptative Hypermedia Authoring) (De Bra \& Stash, 02) que permite convertir en adaptativos toda clase de aplicaciones basadas en la Web, a través de un simple pero poderoso motor de adaptación.

En este artículo vamos a describir un Sistema Hipermedia Adaptativo basado en Web que permite la construcción de cursos adaptativos. Nuestro sistema se ha desarrollado utilizando de base el sistema AHA que como ya hemos comentado es una arquitectura genérica de Sistema Hipermedia Adaptativo que permite crear diferentes tipos de aplicaciones adaptativas. Debido a que AHA es genérico se le han realizado algunas modificaciones para especializarlo a educación, potenciando y añadiendo algunas características. La arquitectura global del sistema AHA está inspirada en el modelo de referencia AHAM (Wu et al. 99), que es una extensión del modelo Dexter (Halasz \& Schwartz, 94). Este modelo fue construido para capturar las estructuras y funcionalidades de los AHS existentes y futuros. En este modelo el sistema está constituido por cuatro partes que trabajan estrechamente unidas: modelo del dominio, modelo del usuario, modelo de 
Construcción de cursos hipermedia adaptativos basados en web utilizando AHA

adaptación y motor adaptativo. El sistema que hemos desarrollado, no sólo desarrolla las funciones que dispone AHA, sino que añade nuevas funciones específicas para entornos educativos que lo hacen innovador. Con la utilización de nuestro sistema se pretende poder realizar la docencia de los cursos que contiene, adaptando toda la información a todos y cada uno de los usuarios que lo ejecuten de forma personalizada, como si de un profesor particular se tratase. En el momento que el usuario entra en el sistema, se guardan sus datos personales y se crea un Modelo de Usuario, que contendrá todas las incidencias que tenga a lo largo de la ejecución del curso. El sistema asignará un nivel al usuario en función de una serie de preguntas, que intentan determinar el conocimiento que éste tiene previa realización de los temas. A partir de aquí, este nivel puede cambiar dependiendo del conocimiento que el usuario adquiera. El sistema realizará un seguimiento del usuario en cada momento, para siempre poder adaptar el contenido y la navegación al nivel que dispone.

La estructura que vamos a seguir en el artículo es: Primero vamos a describir la arquitectura del sistema AHA y las modificaciones que le hemos realizado, después mostraremos un Curso de Linux construido con nuestro sistema, luego veremos la herramienta autor que estamos desarrollando para facilitar la construcción de cursos y finalmente veremos las principales conclusiones y estado actual de nuestro trabajo.

\section{ARQUITECTURA}

La arquitectura interna de un Sistema Hipermedia Adaptativo basado en Web como AHA es muy parecida a la que posee un Sistema Tutor Inteligente, de forma que ambos están formados por (De Bra et. al, 99): un modelo del alumno, un modelo del dominio y un modelo tutor.

\subsection{MÓDULO DE DOMINIO}

El Módulo del Dominio contiene toda la información que se desea enseñar a los alumnos (el qué enseñar). Contiene todos los conocimientos así como la estructura o forma en que éstos se encuentran organizados. Por lo tanto, resulta evidente una labor pedagógica previa, realizada por expertos en la materia que se pretende enseñar. La información del dominio puede ser: textos, imágenes, ejercicios, etc. además de información relativa a importancia, dificultad, relaciones, etc. El objetivo del sistema es que el alumno aprenda todo o la mayor parte del conocimiento representado en el módulo del dominio.

El Modelo de Dominio en el sistema AHA (De Bra \& Calvi, 98) está formado básicamente por conceptos y relaciones entre los conceptos. Un concepto es un 
componente que representa un ítem abstracto de información que es principal para el sistema y que está formado por un identificador, una serie de pares de valores de atributos y un conjunto de enlaces. Un concepto tiene una o varias páginas web relacionadas. Una página está formada por uno o varios fragmentos. Cada página es un fichero XML con fragmentos incluidos condicionalmente y enlaces de hipertexto.

El Modelo del Dominio modificado de AHA que vamos a utilizar está basado en la estructuración típica del material docente de un curso que está formado por una serie de temas que contienen un conjunto de conceptos y estos a su vez pueden contener subconceptos. Por ejemplo un curso podría tener la siguiente estructura (ver Figura 1).

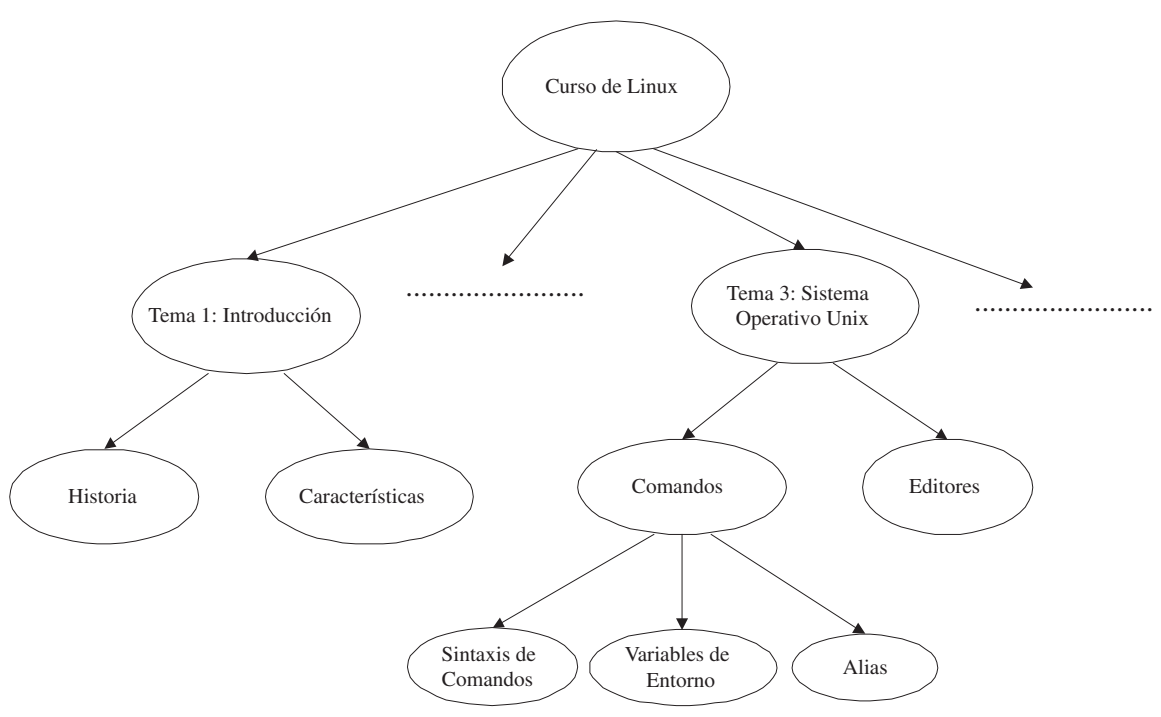

Figura 1. Estructura de un curso ejemplo.

Otra característica importante que se le ha añadido al Modelo del Dominio de AHA es la clasificación o agrupación de los conceptos por niveles de dificultad (principiante, medio y experto), dando lugar que cada tema pueda tener hasta tres niveles de dificultad distintos cada uno con conceptos distintos.

\subsection{MÓDULO DEL ALUMNO}

El Módulo del Alumno o Módulo del Estudiante contiene la información sobre el alumno (a quién enseñar) preferencias, conocimiento, objetivos, historial, etc. Esta información la suele obtener el propio sistema por observación. 
Construcción de cursos hipermedia adaptativos basados en web utilizando AHA

Conforme se le va enseñando al alumno, se van recogiendo datos sobre la forma en la que este aprende (respuestas obtenidas en test, actividades, etc.). Estos datos se utilizan luego para poder ir adaptando el curso a ese alumno en particular. Algunas de las características que se suele recoger en este módulo son: conocimiento del alumno, tipo de aprendizaje que utiliza, preferencias sobre los elementos multimedia utilizados, material didáctico utilizado, historial del alumno, etc.

El Modelo de Usuario de AHA (De Bra \& Calvi, 98) está formado por una serie de atributos asociados a cada uno de los conceptos componentes del Modelo de Dominio. Para cada usuario se mantiene una especie de tabla, en la que se almacena cada concepto con el valor de su atributo correspondiente. El Modelo de Usuario contiene principalmente los atributos nivel de conocimiento (valor entre 0 y 100) que indica el nivel de conocimiento que tiene el alumno en ese concepto y varía conforme el alumno va visitando los distintos conceptos y el atributo leído (valor 0 o 1) que contiene información sobre lo que el usuario ha leído sobre un concepto o un concepto se considera relevante para el usuario.

El Modelo del Alumno modificado de AHA es prácticamente idéntico, con la diferencia en la forma en la que se calcula y los valores que puede tener la variable conocimiento. En nuestro sistema la variable conocimiento se calcula en base a la puntuación obtenida en las preguntas asociadas al concepto al que se refiere y no como en el sistema AHA que simplemente se va incrementando conforme el alumno va leyendo las paginas referentes a los conceptos. Además entre todos los posibles valores entre 0 y 100 sólo puede tomar 4 valores (alto, medio, bajo y nada), donde la correspondencia de valores y significado será:

D 0: significa que no conoce nada. Todavía no ha realizado la pregunta.

1) 10: significa un nivel bajo de conocimiento.

D0: significa un nivel medio de conocimiento.

100: significa un nivel alto de conocimiento.

\subsection{MÓDULO TUTOR}

El Módulo Tutor o Instructor es el motor del sistema o tutor en sí (el cómo enseñar). Se encargada de adaptar la información al alumno en particular, es decir va adaptando el módulo del dominio dependiendo del modelo del alumno. Para ello normalmente suele realizar las siguientes funciones: selecciona el material didáctico que se va ofreciendo (la información que se le va mostrando al alumno dependiendo del nivel de conocimiento que tenga el alumno en un momento determinado), determina la accesibilidad del alumno a los contenidos (dependiendo de todo lo aprendido el alumno tendrá una accesibilidad más o menos amplia de la siguiente información disponible), realiza evaluaciones para poder deter- 
minar el nivel de aprendizaje del alumno se realizan evaluaciones (estas evaluaciones se suelen hacer en base a los resultados obtenidos en ejercicios propuestos al alumno), etc.

La adaptación del contenido de la información de un documento hipermedia y de su estructura de enlaces en el sistema AHA (De Bra et. al, 00) está basada en una serie de reglas. Estas reglas establecerán la estructura de la relación entre el Modelo de Dominio, el Modelo de Usuario y la presentación personalizada que se generará para cada usuario. Las reglas de adaptación específicas deben estar siempre definidas por el autor. De la aplicación de las reglas, obtendremos la adaptación y se presentará al usuario. Las técnicas que usa el sistema AHA para la adaptación son las siguientes:

D Inclusión condicional de conceptos: se refiere a los conceptos que son aconsejables, es decir, conceptos para los que el requerimiento es que una expresión booleana sea verdadera se incluyen en esta página; los conceptos que no sean aconsejables se omiten.

Dcultación o anotación de enlaces: AHA presenta enlaces como texto en colores (no subrayado, como es habitual). Se usan tres tipos de enlaces: buenos serían los enlaces a páginas que son aconsejables y que no han sido leídas (color azul); neutral serían los enlaces a páginas deseadas que ya han sido leídas (color púrpura); y malos serían los enlaces a páginas que no son aconsejables (color negro).

El Modelo del Tutor modificado de AHA se va a realizar también utilizando un conjunto de reglas cuya función es a partir del nivel de conocimiento del alumno, seleccionar los nodos a mostrar, el nivel de dificultad apropiada, y la visibilidad o nodos disponibles. Además en el sistema AHA las reglas aparecían directamente dentro de todos los ficheros del dominio. En nuestro sistema, se han creado unos nuevos ficheros llamados de evaluación de tema en los que se incluyen todas las reglas necesarias para la adaptación de un tema en concreto, de manera que en las páginas XML de contenido sólo habrá contenido, separando de esta forma contenido de adaptación. De forma general la adaptación lo que va a hacer es: si el nivel de conocimiento del alumno en un tema es alto, se le presenta el material de nivel de dificultad alto. Si el alumno aumenta o disminuye su nivel de conocimiento de un tema, se aumentará o disminuirá también la dificultad del material que se le muestra. En la Figura 2 se muestra la adaptación específica de nuestro sistema en un tema de ejemplo.

Como se aprecia en la figura para poder determinar el nivel de conocimiento del alumno en cada tema, primero se realiza un test adaptativo inicial que establece el nivel inicial al cual se le va a mostrar el tema. Después se va obteniendo el progreso mediante las actividades en cada concepto y finalmente se obtiene su nivel final mediante un test clásico final. Para poder finalizar un tema hay que 
Construcción de cursos hipermedia adaptativos basados en web utilizando AHA

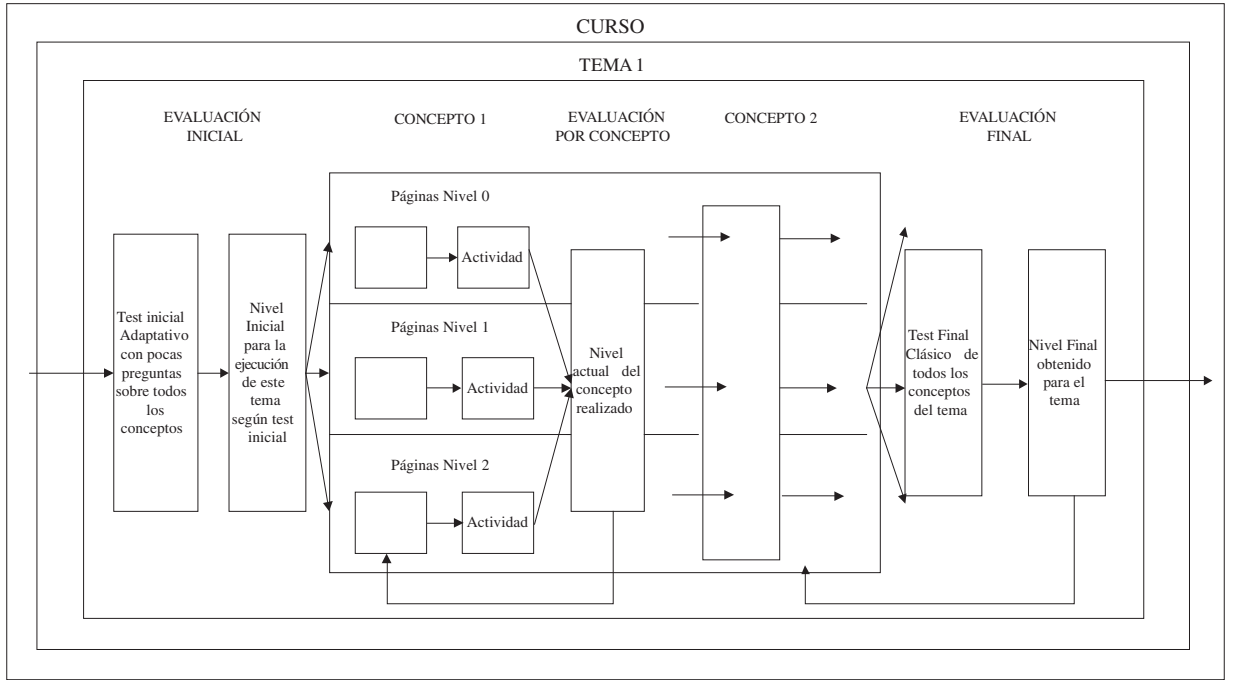

Figura 2. Adaptación de un tema en AHA modificado.

alcanzar previamente nivel experto, es decir, que si inicialmente hemos obtenido un nivel bajo, tendremos que pasar a nivel medio y luego a experto, para poder pasar a otro tema. Tras finalizar un tema, al alumno se le permitirá pasar al siguiente o siguientes temas y todo comenzará de nuevo (primero test inicial, actividades y test final).

\section{CURSO DE LINUX}

Hemos desarrollado un Curso Hipermedia Adaptativo Basado en Web de Linux utilizando nuestro sistema y utilizando multitud de material procedente de otros cursos web, libros (Bandel \& Napier, 00) y revistas (Buelta, 02) relacionadas con Linux. Todo el software que compone nuestro sistema con todo lo necesario para su ejecución (maquina virtual de Java) junto con el Curso de Linux se encuentran disponibles para descargar de forma gratuita en la siguiente dirección Web http://www.uco.es/grupos/eatco/aha. En ella, se nos explica como se hará la instalación, a la vez que nos muestra los enlaces para descargar el software necesario. La instalación es diferente en sistemas Windows que en sistemas Unix. Una vez instalada la maquina virtual de Java sólo hay que descomprir el archivo jswdk.zip y configurar correctamente las variables de entorno (dentro del fichero startserver.bat).

Para poder entrar al sistema, primero hay que arrancar el servidor web, ejecutando el archivo startserver.bat. Entonces, debemos esperar a que nos abra una 


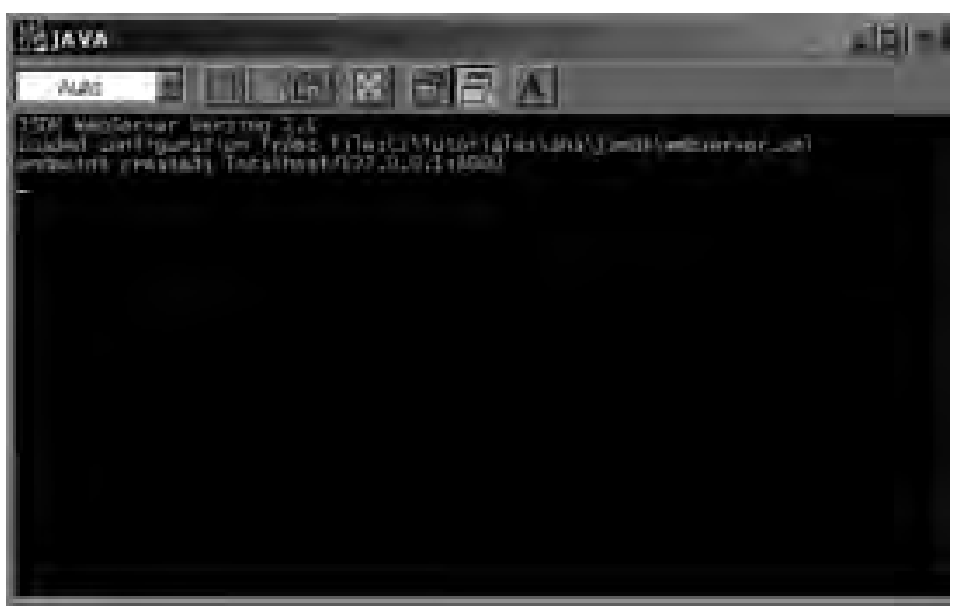

Figura 3. Inicialización del servidor web en el puerto 8000.

ventana MS-DOS donde nos muestra la dirección web y el puerto en el que se publica el curso (ver Figura 3).

Para entrar al curso sólo tenemos que abrir un navegador web y escribir la dirección http://localhost:8000/linux si queremos ejecutarlo en modo local o sustituyendo la palabra localhost por la IP real del ordenador si queremos ejecutarlo de forma remota. Entonces nos aparecerá la pantalla de entrada al sistema (ver Figura 4)

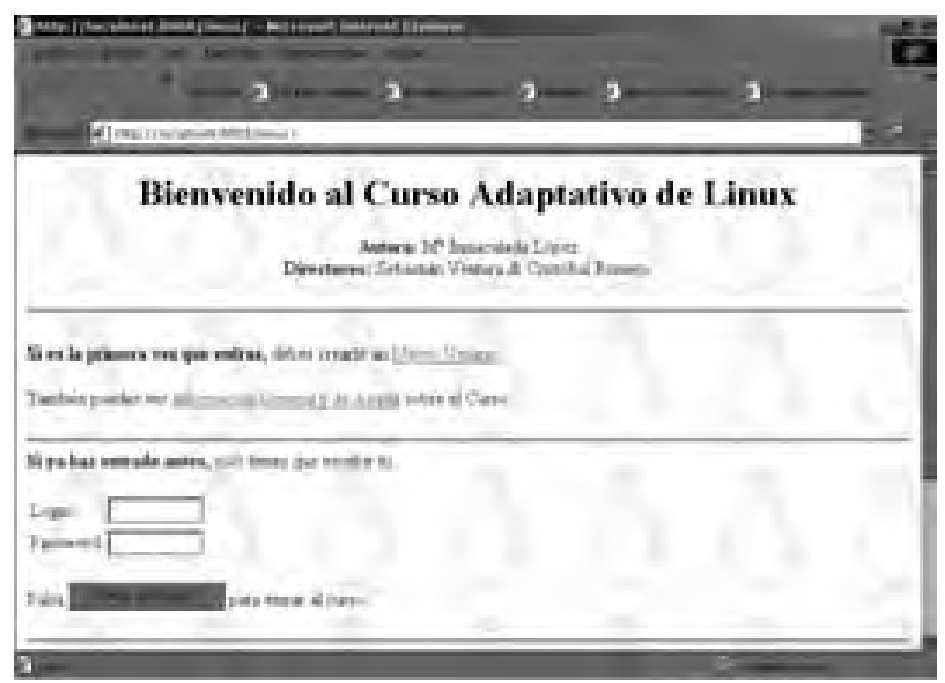

Figura 4. Pantalla Principal de Entrada al Sistema. 
Construcción de cursos hipermedia adaptativos basados en web utilizando AHA

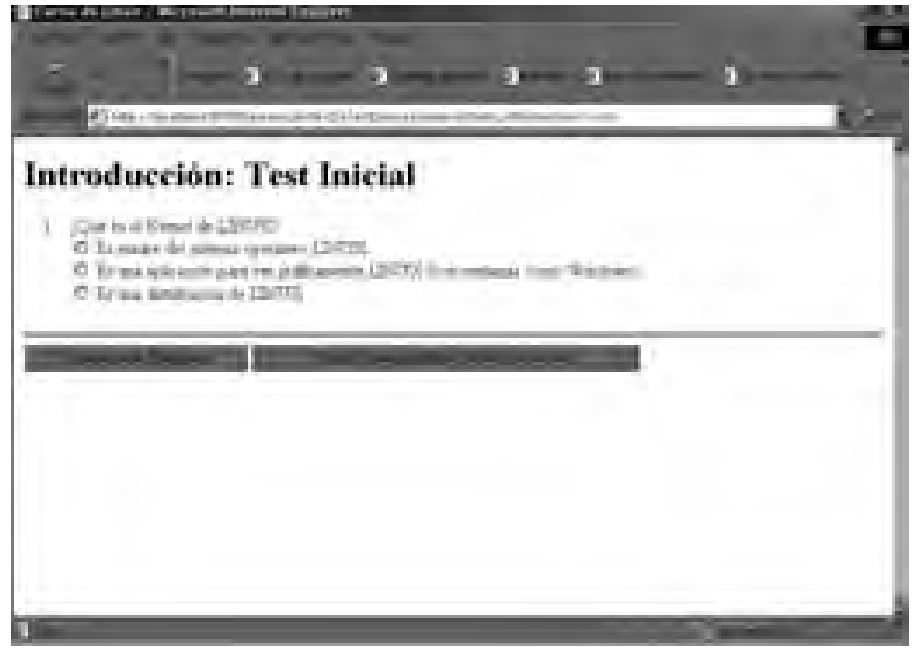

Figura 5. Primera Pregunta del Test Inicial del Primer Tema.

donde se nos pueden presentar dos situaciones distintas. Si es la primera vez que entramos, en la que debemos crearnos un nuevo usuario y las demás veces, que ya disponemos de usuario, por lo que usaremos el que tenemos.

Si ya hemos creado un usuario (no es la primera vez que entramos), solo tenemos que identificarnos en la pantalla principal. Introducimos nuestro login (identificador) y nuestra password (contraseña) y pulsamos en botón Entrar al Curso!. La primera vez que entramos a un nuevo tema del curso, se nos hace un test adaptativo inicial que establecerá el nivel de conocimiento que poseemos antes de realizar el tema. El nivel que obtengamos en este test, será válido para el primer tema. Este Test Adaptativo (Ordoñez, 00) consiste en dependiendo del acierto o fallo de la pregunta actual aumentar o disminuir la dificultad de la siguiente pregunta a realizar. Las preguntas que se nos muestran tienen la apariencia que se muestra en la Figura 5.

Como vemos, es una pregunta tipo test, que nos ofrece tres posibles respuestas de las que sólo habrá una correcta. En todos los tests que se realicen en este curso, siempre habrá una respuesta correcta nada más. Pulsamos con el botón izquierdo del ratón en la que creemos correcta y le damos a Evaluar esta Pregunta. La pantalla siguiente variará en función de nuestro acierto o fallo en la pregunta. Esta primera pregunta tiene un nivel medio, que es siempre el nivel de comienzo. Si acertamos la pregunta, la siguiente que se nos muestra tendrá un nivel alto y si fallamos, tendrá un nivel bajo. Este será el modo de actuar del test, hasta que se realicen las 4 preguntas de que constará. La evaluación de la cuarta 


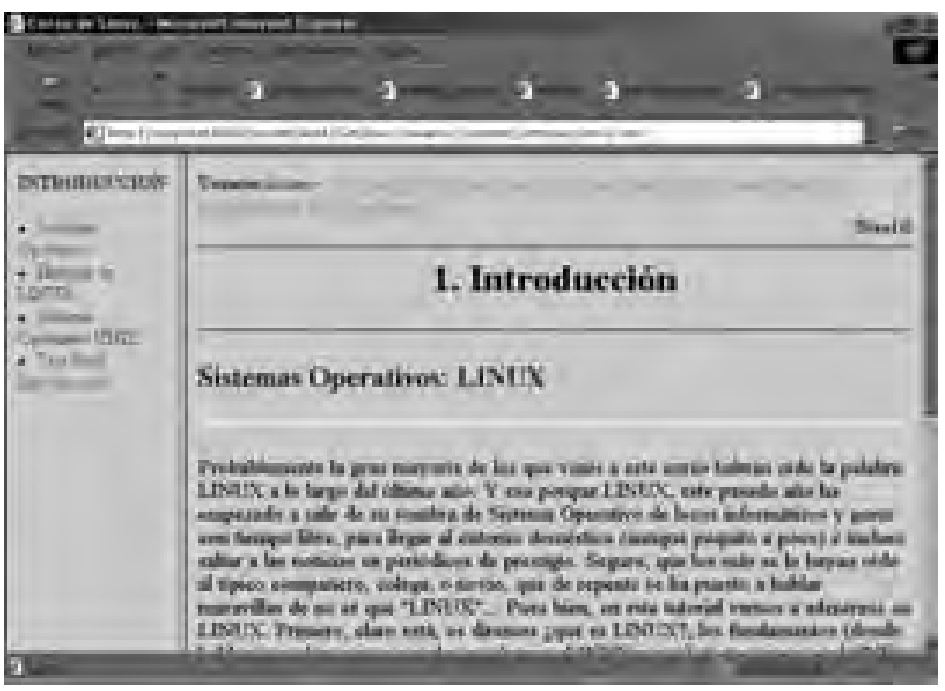

Figura 6. Primer Concepto del Primer Tema con Nivel Bajo.

pregunta del test inicial (que es la última), nos dará paso al tema. Dependiendo del número de preguntas acertadas el sistema nos clasificará dentro de tres niveles de conocimiento (alto, medio o bajo) y nos presentará el tema a ese nivel de conocimiento. Por ejemplo en la Figura 6 se puede ver la primera pantalla del tema 1 con un nivel bajo.

Esta es la pantalla típica del curso y está dividida en dos partes: A la izquierda se muestra el título del tema y todos los conceptos que componen el tema en este nivel junto al test final. A la derecha se muestra en la parte inferior el contenido de cada uno de los conceptos del tema y en la parte superior un menú con información del nombre de usuario y nivel que posee actualmente en el tema, enlaces para ver el conocimiento del tema, conocimiento del curso y desconectarte del curso. El color de fondo de las páginas también indica el nivel que tiene el alumno en cada tema en cada instante. A continuación vamos a describir más detalladamente todas las partes de esta página (Figura 6) y sus significados:

D Índice. A la izquierda de la pantalla, encontramos el índice del tema actual. Nos muestra los conceptos y el test final del tema. Cada uno de estos conceptos son enlaces, de manera que podemos movernos por el tema a nuestro gusto y realizar la lectura de los conceptos en el orden que nosotros queramos establecer. Lo único a lo que nos obliga el curso es tener que realizar las actividades de cada concepto y después de realizar todas las actividades de todos los conceptos de un nivel, realizar el test final para poder pasar a otro nivel o tema diferente. 
Construcción de cursos hipermedia adaptativos basados en web utilizando AHA

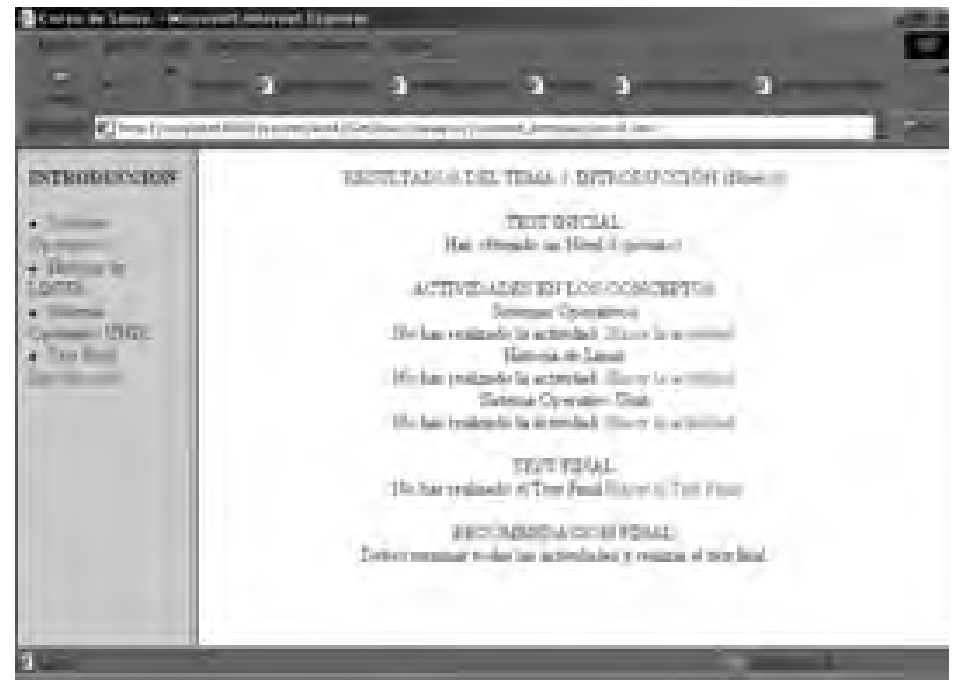

Figura 7. Conocimiento Primer Tema Nivel Bajo.

D Usuario: Nos muestra el identificador del usuario que está realizando el curso.

D Conocimiento Tema Actual. Este enlace nos muestra el conocimiento del tema en el que actualmente estamos. Por este motivo, en el ejemplo de la Figura 6, el enlace se muestra como Conocimiento Tema introducción. Si pulsamos este enlace, nos muestra el conocimiento que hasta ahora hemos obtenido en función de las páginas leídas y las actividades realizadas (Figura 7). Como vemos, lo único en lo que tenemos conocimiento es en el test inicial, que nos dice que obtuvimos un nivel bajo. Puesto que aún no hemos realizado las actividades de los conceptos que componen el tema ni el test final, nos recomienda que hagamos las actividades.

D Conocimiento Curso Linux Completo. En este enlace se nos muestra el índice completo del curso, y los niveles que hemos obtenido en cada uno de ellos. Puesto que el diseño del sistema, se hace para que, aunque se haya obtenido un nivel bajo, se vaya subiendo hasta obtener el nivel experto y así ver todo el curso, el nivel que aparecerá en este índice será el último obtenido, aunque al principio obtuviéramos un nivel más bajo. Como vemos en la Figura 8, hasta ahora no hemos realizado ningún tema. Tan sólo el Tema 1 en el que nos encontramos y en el que aún poseemos el nivel bajo.

Desconectarse del Curso Linux. Con este enlace finalizaremos la sesión del usuario. Con este enlace no desconectamos el curso. Los pasos que seguirán al pulsar el botón. 


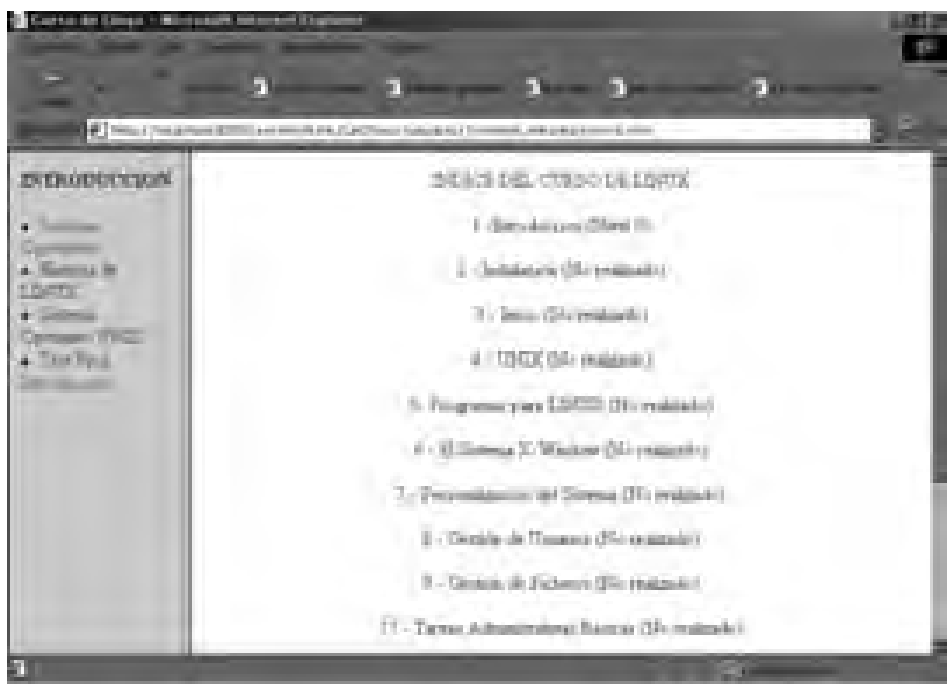

Figura 8. Conocimiento Curso Linux.

Contenido. La parte central e inferior de la página lo ocupa el concepto actual del tema en el que nos encontramos. Muestra la información que se pretende impartir y que el usuario debe leer detenidamente para aprender.

D Actividades. Al final del contenido de cada concepto, en la parte inferior de la página, se encuentra el enlace a la actividad del concepto actual. Una vez leído el concepto, debemos pulsarlo para realizar la actividad que comprobará el conocimiento adquirido en este concepto. La actividad es una pregunta que nos permite evaluar el conocimiento del alumno en ese concepto en particular. Si no se acierta, se volverá a presentar una nueva pregunta. Si se vuelve a fallar se volverá a presentar el contenido del concepto.

Color de Fondo. El color del fondo es una nota a resaltar. Por este color podemos saber en qué nivel nos encontramos en cada tema. Las páginas que se nos muestran con color rosa, son de Nivel Bajo, las de color azul, tienen Nivel Medio y las de color amarillo, tienen Nivel Alto.

Dest Final. El test final que se nos muestra al pulsar en este enlace, es un test clásico que se debe realizar tras haber realizado todas las actividades de los conceptos del tema en ese nivel. Dispone de seis preguntas con tres posibles respuestas y sólo una correcta. Para pasar el test hay que contestar, al menos, tres preguntas correctamente. Dependiendo del número de aciertos se calculará el nivel de conocimiento final que tiene el alumno en ese tema y en ese nivel. La apariencia de un test final se muestra la Figura 9. 
Construcción de cursos hipermedia adaptativos basados en web utilizando AHA

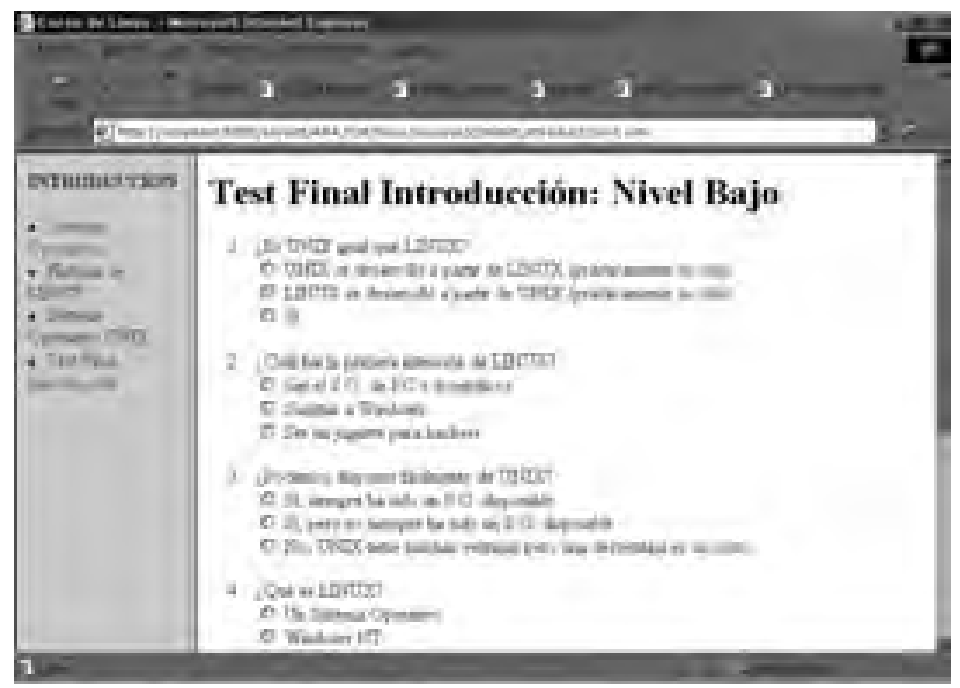

Figura 9. Test Final.

\section{HERRAMIENTA AUTOR}

Para la construcción de un web hipermedia adaptativo utilizando AHA no existe actualmente una herramienta autor completa desde la que se pueda crear, sino que el profesor debe de realizar de forma manual las siguientes pautas (De Bra \& Calvi, 98): Primero debe crear un directorio principal con el nombre del curso dentro del directorio $w w w$ del sistema AHA, que contendrá los siguientes subdirectorios:

D xml. Contendrá las páginas $x m l$ que formen parte de algún modo del curso.

D icons (o images). Contendrá las imágenes del curso.

Dest. Contendrá todos los test del curso.

Además, deben crearse los siguientes archivos en el directorio principal del curso:

docs. Contendrá simplemente una lista de todas las páginas existentes.

Droperties. Describirá la convención de nombres a la que se ha llegado.

Deader y footer. Estos archivos contienen el texto que se introducirán en el encabezado y pie de página de todos los archivos de conceptos.

Dmlgenlist. Será el archivo que contendrá las relaciones que indican como el conocimiento se propaga a través de los conceptos. 
Dmlreqlist. Será el archivo que contendrá las relaciones que indican bajo que condiciones que páginas y conceptos serán aconsejables para el alumno.

Además para la creación de un curso hipermedia adaptativo con nuestra modificación de AHA, se deben tener en cuenta también las siguientes consideraciones.

Donceptos. Todas las páginas, de todos los conceptos y con todos sus niveles, se deben localizar en la carpeta $x m l$ y deben tener la nomenclatura ConceptoTema-Nivel.xml. Los temas y los niveles se indicarán por números y la correspondencia para los niveles será: para el nivel principiante el número 0, para el nivel intermedio el número 1 y para el nivel experto el número 2. Por ejemplo, una página cuyo contenido fuera una introducción del tema 3 con un nivel experto, podría llamarse introduccion3-2.xml.

D Tests Iniciales. Estos tests son adaptativos por lo que hay que crear 3 ficheros distintos uno por cada nivel, se deben localizar dentro del diretorio test y su nomenclatura debe ser testi_TemaNivel.xml. Siendo Tema el nombre del tema y Nivel el nivel al que pertenece. Por ejemplo el test inicial para el tema de programas del nivel bajo, se llamaría testi_programas0.xml. Recordar que hay que crear tres tests distintos, cada uno con un nivel distinto.

D Actividades. Las actividades son preguntas que permiten evaluar un concepto en particular, se deben localizar dentro del diretorio test y su nomenclatura es la exactamente la misma que la del concepto a la que se refiere ConceptoTema-Nivel.xml.

D Tests Finales. Los tests finales serán tests clásicos, se deben localizar dentro del diretorio test y su nomenclatura debe ser testf_TemaNivel.xml. Siendo Tema el nombre del tema y Nivel el nivel al que pertenece este test final. Por ejemplo el test final para el tema de utilidades del nivel medio, se llamaría testf_utilizades1.xml.

D Evaluación. Los ficheros de evaluación contienen todas las reglas necesarias para la adaptación de cada tema a cada alumno en particular dependiendo de su nivel de conocimiento. Debe de existir uno para cada nivel de cada tema, por lo que tendremos tres ficheros de evaluación para cada tema (tres niveles), se deben localizar dentro del diretorio $x \mathrm{ml}$ y la nomenclatura usada para identificarlos debe ser evaluación_TemaNivel.xml. En este archivo se incluirán todas las reglas necesarias para adaptar el contenido del tema, se referirán a valores obtenidos en los test iniciales, test finales y actividades, y deben tener el siguiente formato:

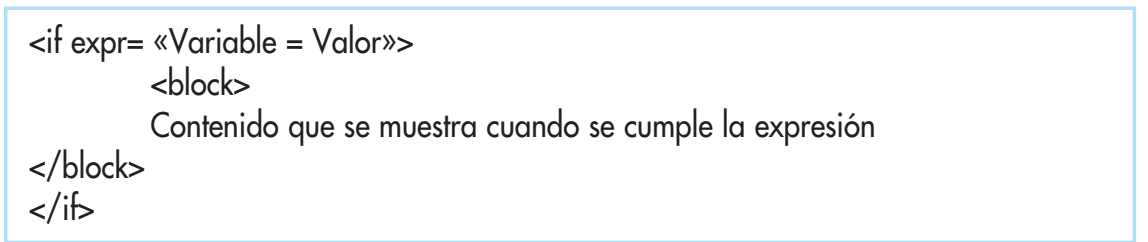




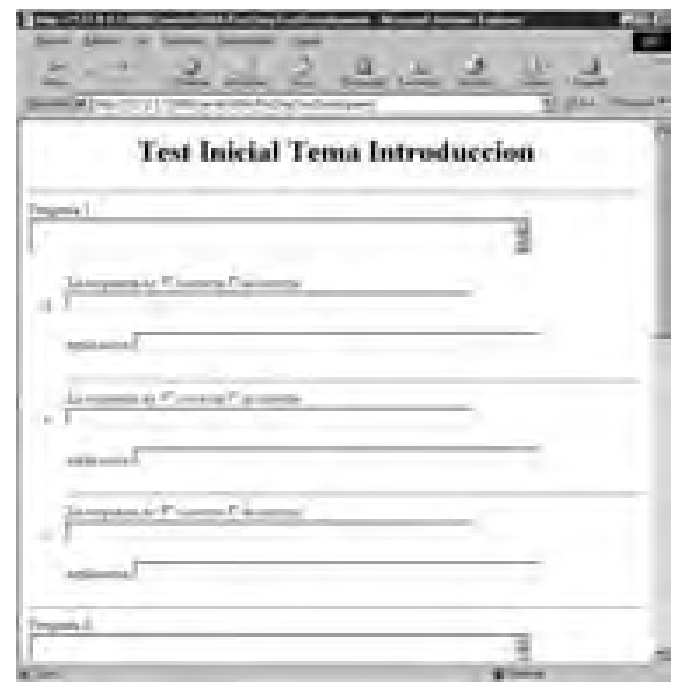

Figura 10. Herramienta para la creación de Test con AHA.

Con respecto a la disponibilidad de herramientas autor para automatizar la creación de web adaptativos, el sistema AHA sólo proporcionando una herramienta para crear los test (Figura 10) y otra para especificar las relaciones de requisitos y de generación (De Bra, 02).

En nuestro sistema estamos desarrollando una herramienta autor completa y específica para nuestro formato de curso adaptativo, con la que el autor del curso pueda construir un curso hipermedia adaptativo desde un interfaz web de una forma fácil e intuitiva y sin la necesidad de escribir código $x \mathrm{ml}$. Para ello el profesor sólo tiene que ir seleccionando las distintas páginas html que forman parte del curso e indicar a que concepto, tema y nivel se refieren (Figura 11) y automáticamente se crearán las páginas $x \mathrm{ml}$ y la estructura del curso.

Para la realización de test iniciales, finales y actividades se puede utilizar la herramienta que ya dispone AHA para creación de test, simplemente teniendo en cuenta la nomenclatura específica que tienen que tener en cada caso. Por último para la creación de los ficheros de evaluación se está desarrollando una herramienta con la que el profesor puede especificar las reglas de adaptación de cada tema sin necesidad de tener que escribir manualmente las reglas.

\section{CONCLUSIONES}

A lo largo de este artículo hemos descrito como se puede construcción un curso hipermedia adaptativo basado en web utilizado de base el sistema AHA 


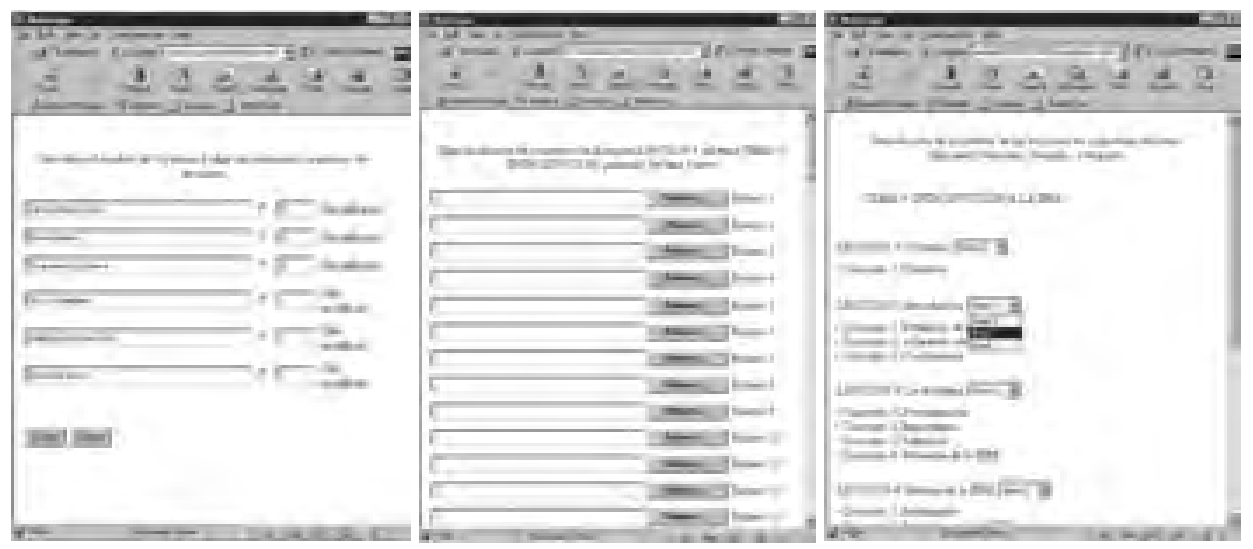

Figura 11. Herramienta para crear los temas de un curso.

(De Bra \& Rutier, 01). El sistema AHA proporciona una arquitectura genérica de Sistema Hipermedia Adaptativo y lo hemos escogido debido a que además de ser un modelo genérico de sistema hipermedia, se dispone del código fuente para poder realizar modificaciones. En concreto se ha modificado parte de la funcionalidad del sistema AHA para especializar su adaptatividad en entornos educativos. Esta ampliación ha consistido principalmente en la incorporación de niveles de dificultad (bajo, medio y alto) de forma, que se pueda controlar que el contenido y la presentación del curso se muestren al nivel de dificultad adecuado para cada alumno en cada instante. También estamos desarrollando una herramienta autor para que el profesor pueda construir un curso adaptativo de forma fácil, cómoda e intuitiva sin necesidad de conocer aspectos internos de implementación.

Hasta la fecha hemos construido varios cursos hipermedia adaptativos basados en web utilizando nuestro sistema: un Curso de Linux (es el primer curso desarrollado, se puede descargar del web junto a nuestro sistema y está siendo utilizado por de alumnos de la Universidad de Córdoba y de ciclos de formación superior en informática también de Córdoba), un Curso de Programación Concurrente (es un curso desarrollado para ser utilizado por los alumnos de la asignatura de Programación Concurrente de Ingeniería Informática de la Universidad de Córdoba) y actualmente estamos comenzando el desarrollo de un Curso de Fortran (curso orientado para alumnos de la asignatura de Programación Científica de Física de la Universidad de Córdoba y que va a ser el primero en construirse utilizando la herramienta autor que estamos terminando).

Nuestra idea actual es poder mejorar los cursos construidos (contenido y estructura) utilizando para ello la información de utilización de los alumnos: tiem- 
Construcción de cursos hipermedia adaptativos basados en web utilizando AHA

pos (utilizados para ver las páginas o contestar preguntas) aciertos (o fallos en las preguntas) y niveles (obtenidos en test iniciales y finales). Toda esta información se recoge en ficheros logs del servidor sobre los que vamos a aplicar técnicas de data mining para descubrir información interesante que pueda servir al profesor para mejorar la efectividad del curso adaptativo (Zaïne, 2001). En concreto estamos trabajando en aplicar algoritmos genéticos para el descubrimiento de reglas de asociación entre los datos de utilización (Romero et al., 2002) estas reglas se le mostrarán al profesor para que decida que cambios puede o debe hacer en el contenido (modificar el texto de una página o pregunta) o estructura del curso (cambiar de nivel un concepto, cambiar de tema un concepto, agrupar varios conceptos, reestructurar los temas, etc). 


\section{REFERENCIAS BIBLIOGRÁFICAS}

Badel, D., Napier, R. (2000) Linux 6. Edición. Prentice Hall.

Buelta, A.G. (2002). Revista Mundo Linux. España.

Brusilovsky, P. (1996) Methods and techniques of adaptive hypermedia. User Modeling and User-Adapted Interaction. Spec Iss. On Adaptive Hypertext and Hypermedia. 6 (2-3), 87-129.

Brusilovsky, P. (1999) Adaptative Educational Systems on the World-Wide-Web: A Review of Available Technologies.

De Bra, P. Calvi, L. (1998). AHA: a Generic Adaptive Hypermedia System. $2^{\text {nd }}$ Workshop on Adaptive Hypertext and Hypermedia. Pp. 1-10.

De Bra, P. We, H., P., Aerts, A., Houben, G.J. (2000) Making General-Purpose Adaptative Hypermedia Work Procedings of the WebNet Conference, pp. 117-123.

De Bra, P. Rutier, J.P. (2001) AHA! Adaptive Hipermedia for All. Proceedings of the WebNet Conference, pp. 262-268.

DE BRA, P., (2002) Adaptive Educational Hypermedia on the Web. Communications of the ACM, vol. 45 , nr. 5, pp. 60-61.

De Bra, P., Stash, N. (2002) AHA! A General-Purpose Tool for Adaptive Websites. World Wide Web Conference, Poster Session.

Halasz, F., Schwartz, M. (1994) The Dexter Hypertext Referente Model. Communications of the ACM. 37:2, pp. 30-39.

ORDóÑEz, T. (2000). Diseño y evaluación de un sistema de administración de test por ordenador (SEA: Sistema de Evaluación Asistida). Tesis Doctoral. Valencia.

Romero, C., De Castro, C., Ventura, S. (2002). Using Genetic Algorithms for Data Mining in Web-based Educational Hypermedia System. Workshop on Adaptive Systems for Web-based Education. Malaga.

Wu, H., Houben, G.J., De BRA, P. (1999) AHAM: A Dexter-based Reference Model for Adaptive Hypermedia, Proceedings of the ACM Conference on Hypertext and Hypermedia, pp. 147156, Darmstadt, Germany.

Zaïne, O. R. (2001) Web Usage Mining for a Better Web-Based Learning Environment. Technical Report.

\section{PERFIL ACADÉMICO Y PROFESIONAL DE LOS AUTORES}

Cristóbal Romero Morales: Becario Docente de la Junta de Andalucía en el departamento de Informática y Análisis Matemático de la Universidad de Córdoba.

Líneas de investigación: Sistemas Hipermedia Adaptativos, Sistemas Tutores Inteligentes, Data Mining.

E-mail:ma2romoc@uco.es

Sebastián Ventura Soto: Profesor Titular de la E. P. S. de la Universidad de Córdoba.

Líneas de investigación: Algoritmos Evolutivos, Sistemas Hipermedia Adaptativos, Data Mining.

E-mail: sventura@uco.es

Carlos de Castro Lozano: Profesor Titular de la E. P. S. de la Universidad de Córdoba.

Líneas de investigación: Sistemas Multimedia, Enseñanza a distancia, Tecnologías Adaptativas.

E-mail: cdecastro@uco.es

Departamento de Informática y Análisis Numérico.

Campus Universitario de Rabanales.

Ctra. Madrid-Cádiz, Km. 396,2 14071 Córdoba

Teléfono: +34 57218630 Fax: +34 57218630 